Polymer Journal, Vol. 4, No. 4, pp 351-357 (1973)

\title{
Photosensitive Ionomer. VI. Image Formation by the Use of Mercurous Copoly(vinyl alcohol-maleate)
}

\author{
Kazuyuki Sugita, Hisashi Muroga, and Shin SuzukI \\ Department of Graphic Engineering, Faculty of Engineering \\ Chiba University, 1-33, Yayoi-cho, Chiba, Japan.
}

(Received March 30, 1972)

\begin{abstract}
The sensitivity of light-sensitive material depends both on the quantum yield of the photochemical reaction and on the property change (e.g., absorbance, solubility, and so on) which is brought about by the reaction. The sensitivity of photopolymers of the photopolymerization type is improved by increasing the latter factor. Improvements of photopolymers of the photocrosslinking type may be possible by increasing the former factor.

A photosensitive ionomer, a polymer which on exposure crosslinks through metallic ions of variable valence, was prepared. A photosensitive plate coated with the ionomer formed a visible and relief image on irradiation, and the latent image was intensified by treating with a reducing or a oxidizing developer.

Mercurous copoly(vinyl alcohol-maleate) was prepared as an example of a photosensitive ionomer and the image-forming properties were compared with those of mercurous copoly(vinyl alcohol-acrylate) and mercurous copoly(vinyl alcohol-methacrylate) which were previously reported. Mercurous copoly(vinyl alcohol-maleate) was found to be the most sensitive for forming a visible image, but the least sensitive for a relief image. It was considered that the structure of this ionomer, in which two mercurous carboxylate groups were adjacent, was suitable to the photochemical reaction, but the reaction did not bring about enough property change to form a relief image.
\end{abstract}

KEY WORDS Improvement of Photosensitivity / Quantum Yield / Property Change Brought about by the Photochemical Reaction / Enlargement of Apparent Quantum Yield / Photocrosslinking Reaction / Photosensitive Ionomer / Mercurous Copoly(vinyl alcohol--maleate) / Disproportionation of Mercurous Ion / Structure of Mercurous Polymeric Carboxylate /

The photopolymers, which undergo chemical reaction on short-term irradiation and are available for image formation, are divided into three types: photolysis, photopolymerization, and photocrosslinking. ${ }^{1}$ Photopolymers of the first and the third types are functional polymers, and the photochemical reaction is a polymer reaction in the solid phase.

An example of the photopolymer of the first type is diazo resin. When the polymer is exposed to an adequate light source, the chemical reaction of side groups occurs and brings about a change of the surface property, e.g., hydrophilic into hydrophobic, or vice versa. The photopolymers of the second and the third types form relief images, by taking advantage of the change of solubility towards solvents. Photopolymerization types form an insoluble polymer through chain-propagation polymerization, with irradiation only participating in the initial step to initiate the reaction. However, the change of solubility is not large enough to form a relief image, because the solubility does not decrease in proportion to the increase of molecular weight. The property change per photoinduced reaction is thus comparatively small. A photopolymer of the pure photopolymerization type swells even after it has been hardened by irradiation, and has little importance as an image-forming material. In order to eliminate 
the swelling character, bifunctional olefin is mixed with the monofunctional monomer, and the photopolymer actually used for making a letter-press plate is partly of the photocrosslinking type. This is considered to be an improvement of the sensitivity by increasing the property change per photoinduced reaction.

On the other hand, a photocrosslinking reaction proceeds with difficulty, for it is a reaction between solid polymers of large molecular weight. In spite of the small quantum yield of the photocrosslinking reaction, photopolymers of the third type are sensitive enough to be available for image formation. The fairly large photosensitivity may be due to a steep decrease of the solubility caused by the photocrosslinking reaction. This phenomenon will be easily understood if we recall the fact that a polymerization mixture becomes gradually viscous and then, suddenly, gelation takes place in the polycondensation system. As the property change per photocrosslinking reaction is large, a method of further improvement of the photosensitivity cannot be considered possible except by increasing the apparent quantum yield.

In silver halide photography, metallic silver, formed by the photoreduction of silver halides, is intensified in the reducing development process, and the apparent value of quantum yield is very large. The present photopolymer of the photocrosslinking type forms crosslinkages of covalent bonds, and there seems to be no possibility of increasing the apparent quantum yield by such after-treatment as corresponds to the development of silver halide. If the crosslinkages are ionic, a treatment with oxidizing agents will increase the number and/or strength of the ionic crosslinkages and may result in an increase of the apparent value of quantum yield. Therefore, the authors examined the effects of treatment with oxidizing agents on ionically crosslinked polymer.

It is well known that mercurous acetate disproportionates to mercuric acetate and metallic mercury. ${ }^{2}$ A photosensitive ionomer was prepared by applying the disproportionation to mercurous salts of polymeric carboxylate. The authors have already prepared two examples of photosensitive ionomers, ${ }^{3,4}$ and reported the image formation by the photochemical reaction, the reducing development of a latent image to a visible image,,$^{3,4}$ and the oxidizing development of a latent image to a relief image. ${ }^{5}$ The polymer, as a reactant, contributes to all these processes. The influences of molecular weight and the content of photosensitive groups on the image-forming properties were discussed in the previous paper. ${ }^{3}$ In this paper, mercurous copoly(vinyl alcohol-maleate) was prepared as another example of the photosensitive ionomer. In addition to a comparison of the image-forming properties with those of mercurous copoly (vinyl alcohol-acrylate) and mercurous copoly (vinyl alcohol-methacrylate), it is a purpose of this paper to discuss in more detail the factors which may control the photochemical reaction of polymers in the solid phase.

\section{EXPERIMENTAL}

\section{Materials}

Maleic anhydride was purified by recrystallization from chloroform. Vinyl acetate and xylene were purified by distillation of commercially available reagents. Other reagents used were of extra pure grade.

\section{Apparatus}

Titration were performed by using a Yokokawa $\mathrm{pH}$ meter KPH-51B. The wedge spectrogram was obtained with an Ushio xenon arc lamp UXL-500D and a Narumi monochromator RM-23. Photosensitive plates were irradiated by an Ushio low-pressure mercury lamp ULI-309 (a chemical lamp of 6W) from a distance of $15 \mathrm{~cm}$.

\section{Preparation of Mercurous Maleate and Photo- sensitive Plates}

A 10-\% aqueous solution of sodium hydroxide was exactly neutralized by a $41-\%$ aqueous solution of maleic acid. A mercurous nitrate solution, the concentration of which was previously determined by titration with $0.1-N$ potassium hydroxide, was added to the sodium maleate solution with vigorous stirring in the dark, and mercurous maleate was precipitated as a white flake. In this treatment the volume of the mercurous nitrate solution was controlled so that the amount of nitrate ion in the mercurous 
nitrate solution was equimolar to that of sodium ion in the sodium maleate solution. The white flakes were filtered, washed with water, and uniformly dispersed in a $20-\%$ aqueous solution of poly(vinyl alcohol). The emulsion was spread over glass plates and left to dry.

Preparation of Copoly(vinyl alcohol-maleic acid)

This copolymer was prepared by referring to the method used to obtain copoly(vinyl alcohol-acrylic acid) and to the results of the photosensitive plate made from it. $^{3}$ Vinyl acetate, maleic anhydride, benzoyl peroxide and xylene of the quantity indicated in Table $I$ were placed in a tube (diameter, $2.5 \mathrm{~cm}$; length, 11 $\mathrm{cm})$. After evacuating the air, the tube was sealed off under reduced pressure in nitrogen. The tube was heated in a water bath kept at $80^{\circ} \mathrm{C}$ and polymerization was allowed to proceed at this temperature for $1 \mathrm{hr}$. The polymerization products were dissolved in $90 \mathrm{ml}$ of boiling methanol in a 200-m $l$ flask equipped with a condenser. Five per cent sodium methoxide in methanol was added to the refluxing polymer solution in $1-\mathrm{m} l$ portions at intervals of $5 \mathrm{~min}$. Ten $\mathrm{m} l$ of the solution were sufficient to catalyze the methanolysis. After addition of the sodium methoxide solution, refluxing was continued for further $15 \mathrm{~min}$ and the flask was left to cool. Ninety $\mathrm{m} l$ of ethyl ether were added to the flask for complete precipitation of the polymer. The precipitates were separated by decantation and centrifugation at $5000 \mathrm{rpm}$, washed several times with a mixed solvent of methanol and ethyl ether ( $1: 1$ by volume) and then dried. The ratio of maleic acid incorporated in the copolymer was determined by titrating carboxyl groups. Molecular weight was estimated relatively by measuring a reduced viscosity, $\eta_{\mathrm{sp}} / c$. As the correlation curve between $\eta_{\mathrm{sp}} / c$ and $c$ was not a straight line, intrinsic viscosity [ $\eta]$ could not be determined. The maleic acid content and the reduced viscosity of each copolymer is indicated in Table I.

\section{Preparation of Mercurous Copoly(vinyl alcohol- maleate) and Photosensitive Plates}

One $\mathrm{g}$ of copoly(vinyl alcohol-maleic acid) was dissolved in $50 \mathrm{~m} l$ of water and exactly neutralized by $0.1-N$ sodium hydroxide. Under vigorous agitation in the dark, the mercurous nitrate solution was added to the sodium copoly(vinyl alcohol-maleate) solution. The solution became a milky white emulsion. In preparing mercurous copoly (vinyl alcohol-acrylate), too much mercurous acrylate content decreased the film-forming ability of the copolymer, and too small an amount of mercurous acrylate component gave poor sensitivity to the layer. ${ }^{3}$ The carboxyl group of the copolymer was partly mercurized. In this case the molar ratio of the mercurized carboxyl group to the unmercurized one was determined, without regard for the maleic acid content in the copolymer, after some preliminary

Table I. Monomers ${ }^{a}$ and characterization of copoly(vinyl alcohol-maleic acid)

\begin{tabular}{ccccccc}
\hline $\begin{array}{c}\text { Copolymer } \\
\text { number }\end{array}$ & $\begin{array}{c}\text { Maleic anhydride, } \\
\mathrm{g}\end{array}$ & $\begin{array}{c}\text { Vinyl acetate, } \\
\mathrm{g}\end{array}$ & Xylene, $\mathrm{g}$ & Yield, wt\% & $\begin{array}{c}\text { Maleic acid in } \\
\text { copolymer, wt } \%\end{array}$ & $\eta_{\mathrm{sp} p} / c^{\mathrm{b}}$ \\
\hline 201 & 0.2 & 9.8 & 1.0 & 72.9 & 3.1 & 0.23 \\
202 & 0.2 & 9.8 & 2.0 & 72.2 & 3.1 & 0.22 \\
205 & 0.2 & 9.8 & 5.0 & 52.3 & 5.1 & 0.16 \\
210 & 0.2 & 9.8 & 10.0 & 41.2 & 5.1 & 0.12 \\
501 & 0.5 & 9.5 & 1.0 & 70.5 & 7.1 & 0.54 \\
502 & 0.5 & 9.5 & 2.0 & 67.9 & 6.6 & 0.41 \\
505 & 0.5 & 9.5 & 5.0 & 57.7 & 8.0 & 0.30 \\
510 & 0.5 & 9.5 & 10.0 & 40.7 & 9.9 & 0.22 \\
1001 & 1.0 & 9.0 & 1.0 & 53.7 & 18.2 & 0.39 \\
1002 & 1.0 & 9.0 & 2.0 & 52.6 & 20.2 & 0.32 \\
1005 & 1.0 & 9.0 & 5.0 & 60.2 & 15.1 & 0.23 \\
1010 & 1.0 & 9.0 & 10.0 & 58.5 & 16.4 & 0.17 \\
\hline
\end{tabular}

a $0.1 \mathrm{~g}$ of benzoyl peroxide was used as an initiator in each case.

b Measured at $30^{\circ} \mathrm{C}$ on an aqueous solution of the copolymer $(0.25 \mathrm{~g} / 100 \mathrm{~m} l)$. 


\section{K. Sugita, H. Muroga, and S. Suzuki}

Table II. Image-forming properties of mercurous copoly(vinyl alcohol-maleate) plate

\begin{tabular}{|c|c|c|c|c|c|c|}
\hline $\begin{array}{l}\text { Copolymer } \\
\text { number }\end{array}$ & $\begin{array}{l}\mathrm{Hg} \text { maleate } \\
\text { in copolymer }\end{array}$ & $\begin{array}{l}\text { Film } \\
\text { formation }^{b}\end{array}$ & Photosensitivity $^{c}$ & $\begin{array}{c}\text { Reducing } \\
\text { development }\end{array}$ & Fixation $^{b}$ & $\begin{array}{c}\text { Oxidizing } \\
\text { development }\end{array}$ \\
\hline 201 & 4.1 & 0 & 22 & 3 & $x$ & $\times$ \\
\hline 202 & 4.1 & 0 & 12 & 7 & $x$ & $x$ \\
\hline 205 & 4.3 & $\triangle$ & 20 & 5 & $x$ & $\times$ \\
\hline 210 & 4.1 & $\triangle$ & 20 & 6 & $x$ & $x$ \\
\hline 501 & 6.1 & $\bigcirc$ & 10 & 3 & $x$ & $x$ \\
\hline 502 & 7.6 & 0 & 10 & 3 & $\times$ & $x$ \\
\hline 505 & 11.5 & 0 & 3 & 3 & $x$ & $x$ \\
\hline 510 & 11.5 & $\triangle$ & 10 & 8 & $x$ & $x$ \\
\hline 1001 & 9.5 & $\triangle$ & 10 & 10 & $x$ & $x$ \\
\hline 1002 & 8.8 & 0 & 5 & 5 & $x$ & $x$ \\
\hline 1005 & 9.5 & $\triangle$ & 7 & 7 & $x$ & $\times$ \\
\hline 1010 & 15.5 & $\triangle$ & 7 & 7 & $\times$ & $\times$ \\
\hline PVA-AA ${ }^{\circ}$ & $6.8-10.0$ & 0 & $20-30$ & $0.5-1$ & 0 & 0 \\
\hline PVA-MAA $^{\mathrm{f}}$ & $13.2-15.0$ & 0 & $30-50$ & $1-2$ & $\triangle$ & $x$ \\
\hline
\end{tabular}

a Calculated values in wt $\%$.

b The abbreviations are explained in the text.

c Exposure duration in min, required for the formation of a print-out image.

d Exposure duration in $\mathrm{min}$, required for the formation of a developable latent image.

e Copoly(vinyl alcohol-acrylate).

f Copoly(vinyl alcohol-methacrylate).

trials to obtain an excellent photosensitive layer. The mercurous salt of the copolymer was separated by centrifugation at $10000 \mathrm{rpm}$. The quantity of mercurous ions in the mother liquid was too small to be titrated. Mercurous ions in the nitrate solution were thus regarded as being nearly quantitatively fixed to side chains of the copolymer. The mercurous maleate content in mercurous copoly(vinyl alcoholmaleate) was calculated and is shown in Table II. The mercurous salt precipitated was again uniformly dispersed in a small quantity of water, spread over glass plates, and left to dry.

\section{RESULTS}

\section{Photographic Characteristics of Mercurous Maleate Plates}

Prior to the study of image-forming properties of the copolymer, photographic characteristics were examined on a photosensitive emulsion consisting of mercurous maleate and poly(vinyl alcohol). This was regarded as a model of the mercurous copoly(vinyl alcohol-maleate) emulsion.

The wedge spectrogram showed that the plate was sensitive to light of $250-350 \mathrm{~nm}$, with the

Table III. Photographic characteristics of mercurous monomeric carboxylate plate

\begin{tabular}{|c|c|c|c|c|}
\hline & Photosensitivity ${ }^{\mathrm{a}}$ & $\begin{array}{c}\text { Reducing } \\
\text { development }\end{array}$ & $\begin{array}{l}\text { Photosensitive } \\
\text { region }\end{array}$ & $\underset{\text { increase }^{c}}{\text { Maximum density }}$ \\
\hline Mercurous maleate & 10 & 1 & $250-350 \mathrm{~nm}$ & 1.0 \\
\hline Mercurous acrylate & 20 & 1 & $250-340 \mathrm{~nm}$ & 0.6 \\
\hline Mercurous methacrylate & $(30)^{d}$ & $-^{d}$ & $-^{\mathrm{d}}$ & $-^{d}$ \\
\hline
\end{tabular}

a Exposure duration in $\mathrm{min}$, required for the formation of a print-out image.

b Exposure duration in $\mathrm{min}$, required for the formation of a developable latent image.

c Measured by a printing-out method.

d Could not be measured accurately because of heavy fogging. 
white photosensitive layer changing dark brown. The plate was placed in a dark box, irradiated stepwise by changing the exposure duration, and the reflection density was measured. Density was plotted against exposing time. The curve was S-shaped. The maximum density was $c a .1 .0$ above the fog. The exposed plate was immersed in an aqueous alcoholic solution (water : alcohol $=1: 4$, by volume) of hydroquinone. In a few minutes, the part of plate irradiated for $0.5-500 \mathrm{~min}$ become dark grey, and development was observed. Fogging was found on the unexposed part after development for longer than $20 \mathrm{~min}$. Some of results are indicated in Table III.

\section{Image-forming Properties of Mercurous Copoly- (vinyl alcohol-maleate) Plates}

The emulsion was white and milky, but it dried down to a transparent photosensitive layer. The layer made from Copolymer 1001 (see Tables I and II) peeled off from the glass plate, when it dried. The wedge spectrogram (obtained by a printing-out method) showed that the layer colored dark brown on exposure to the light ranging from $250 \mathrm{~nm}$ to $310 \mathrm{~nm}$. The plate was irradiated stepwise by a low-pressure mercury lamp for $20,10,5,2,1$ and $0 \mathrm{~min}$, respectively, and the color change of the layer was investigated. The exposed plate was developed in an aqueous alcoholic solution of hydroquinone for $10 \mathrm{~min}$, and the color change was investigated again. In the reducing development, the photosensitive layer made from copolymers 205, 210, 510, 1005, and 1010 swelled and the image was disorted. The exposure durations, which were required for the formation of a print-out image and a developable latent image, were measured and are cited in Table II.

The exposed plate was placed in a fixing solution composed of acetic acid $(10-15 \mathrm{ml})$, water $(10-15 \mathrm{ml})$ and alcohol $(80-70 \mathrm{~m} l)$. The photosensitive layer, both the exposed part and the unexposed part, swelled and became detached from the plate. The print-out image could not be fixed by this procedure. Treatment of the exposed plate with a potassium permanganate solution $\left(0.05 \mathrm{~g}\right.$ of $\mathrm{KMnO}_{4}$ in $30 \mathrm{ml}$ of acetic acid and $70 \mathrm{ml}$ of water) did not give a relief image, either. This means that the oxidizing development was unsuccessful on mercurous copoly (vinyl alcohol-maleate) plates. The imageforming properties are summarized in Table II.

\section{DISCUSSION}

\section{Molecular Weight of Photosensitive Ionomer}

The influence of molecular weight on the properties of the photosensitive layer was already considered in the previous paper, which dealt with mercurous copoly(vinyl alcohol-acrylate). ${ }^{3}$ When the molecular weight was too large, the layer was not transparent, but partly white and turbid. This was ascribed to strong intermolecular force which prevented mercurous carboxylate groups from dispersing homogeneously in polymer segments. If the molecular weight was too small, the image on the plate made therefrom showed a swelling character even after it had been hardened by irradiation. Therefore, the copolymer must be within a definite range of molecular weight. Moderate strength of the intermolecular force may be essential to the film-forming and the image-forming abilities.

Referring to the results mentioned above, copoly(vinyl alcohol-maleic acid) was prepared from the monomers indicated in Table I. When the parent copolymer was of smaller molecular weight, the layer swelled in the reducing development. Copolymer 1001, which may be regarded as having comparatively large molecular weight, did not give an excellent photosensitive layer on account of its poor adhesion to the glass plate. Copolymer 1002 was the best among four copolymers prepared from $1.0 \mathrm{~g}$ of maleic anhydride and $9.0 \mathrm{~g}$ of vinyl acetate. The copolymers of larger molecular weight gave good photosensitive layers in two series of copolymers 201,202, 205, and 210, and copolymers 501, 502, 505, and 510. If the molecular weight were much larger, the copolymer might form partly white and turbid layer. From the copolymers investigated here, an optimum condition of molecular weight could not be observed.

\section{Comparison of the Image-forming Property}

For the photosensitive layer of mercurous copoly(vinyl alcohol-acrylate), 30-, 5-, and 1min exposures were sufficient to form a print- 


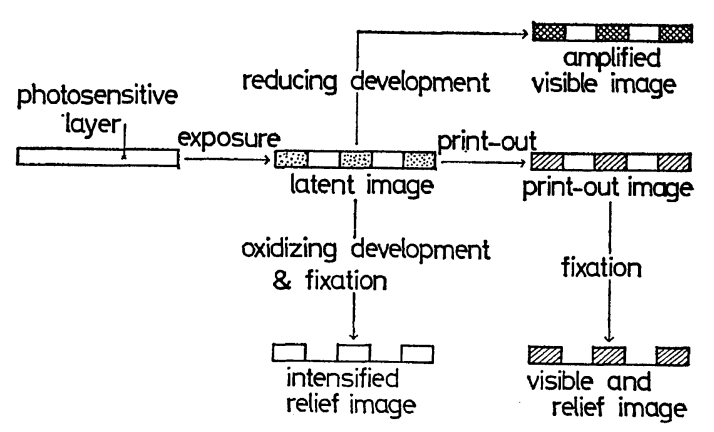

Figure 1. Image-forming processes by the use of mercurous copoly(vinyl alcohol-acrylate).

out image, a latent relief image, and a latent visible image, respectively. The print-out image was fixed by treating with a mixed solvent of acetic acid, water and alcohol. The latent image was converted to a visible image by reducing development, or to a relief image by oxidizing development and fixation. ${ }^{3,5}$ (see Figure 1) The photosensitive layer of mercurous copoly(vinyl alcohol-methacrylate) gave a print-out image and a latent visible image by exposing for 50 min and $2 \mathrm{~min}$, respectively. Though the latent image was successfully amplified by reducing development, fixation did not produce so complete a relief image as that of mercurous copoly (vinyl alcohol-acrylate). Oxidizing development was unsuccessful in this case. ${ }^{4}$ The image-forming properties of mercurous copoly (vinyl alcohol-acrylate) and copoly(vinyl alcohol-methacrylate) are added to the data in Table II to be compared with those of mercurous copoly(vinyl alcohol-maleate).

Mercurous copoly(vinyl alcohol-maleate) formed a visible image by a printing-out method or by reducing development of a latent image. The exposure duration, required for the formation of a print-out image, was shorter than that of the other ionomers previously studied. It may be possible to conclude that mercurous copoly(vinyl alcohol-maleate) is more sensitive to light. A relief image could not be formed on the photosensitive plate by fixation of a print-out image or by oxidizing development and fixation of a latent image. The change of solubility brought about by the photochemical reaction is assumed to be too small to form a relief image.
Intrinsic Absorption of Polymeric Carboxylic Acid In the previous paper, failure to form a relief image in oxidizing development was ascribed to the light-absorbing character of the parent copolymer. ${ }^{4}$ When a mercurous copoly(vinyl alcohol-methacrylate) plate with a latent image was immersed in a potassium permanganate solution, the surface of the photosensitive layer turned insoluble, but the inner part of the layer was still soluble. The reason was considered to be absence of development centers in the inner part. The absorption spectrum of the parent copolymer in the near ultraviolet region, overlaps the photosensitive region of the mercurous salt, and supports the contention that light does not penetrate deeply into the photosensitive layer and induce disproportionation of mercurous ion there.

Copoly(vinyl alcohol-maleic acid) absorbs light in the near ultraviolet region more strongly than copoly(vinyl alcohol-methacrylic acid). However, the surface of the photosensitive layer, on which a print-out image was formed, remained soluble in the fixing solution. Mercurous copoly(vinyl alcohol-maleate) was considered to be more sensitive to light, and, therefore, the intrinsic absorption of the parent copolymer seems not to be the main reason for the failure to form relief images in oxidizing development and fixation.

\section{Structure of Mercurous Carboxylate Group}

It may be more reasonable that the difference between the image-forming abilities depends on the structural aptitude of mercurous carboxylate groups incorporated in the copolymer chain. The mutually adjacent structure of two mercurous carboxylate groups, probably, causes the larger sensitivity in forming a visible image, and, at the same time, the smaller sensitivity in obtaining a relief image. The exposure duration required for the formation of a print-out image is the shortest of the three ionomers. This fact may suggest that the structure of mercurous maleate is suitable for the disproportionation of the mercurous ion to mercuric ion and metallic mercury, and the quantum yield of the photochemical reaction may be large. The mercuric ion forms a crosslinkage between two carboxylate groups, however, the crosslink- 
age is not intermolecular but mainly intramolecular. The crosslinkages do not contribute to the change of solubility of the copolymer chains enough to form a relief image. The exposure duration, required for the formation of a developable latent image was not much shorter than that of the other ionomers. The structure may be indifferent to the reduction of mercurous ion to metallic mercury, which proceeds in the reducing development.

Further support of these considerations is found among the photographic characteristics of mercurous salts of monomeric carboxylic acids (see Table III). The photosensitivity and the maximum density increase of the mercurous maleate plate were larger than those of the mercurous acrylate plate. The exposure duration which was required to form a developable latent image on a mercurous maleate plate, was as long as that of a mercurous acrylate plate. These results also indicate that the structure of mercurous maleate is favorable particularly for the disproportionation of mercurous ion to mercuric ion and metallic mercury.

In conclusion, it may be possible to summerize that there are four factors which control the photochemical reaction and the image-forming ability of the photosensitive ionomer. They are as follows.

1. Molecular weight of the ionomer

2. Content of photosensitive groups in the ionomer

3. Intrinsic absorption of polymeric carboxylic acid

4. Structure of the photosensitive group (mer- curous carboxylates)

The last factor has complicated effects both on the photochemical reaction and on the imageforming ability. The structure with two adjacent mercurous carboxylate groups is suitable for the photochemical disproportionation of mercurous ion, but it screens the change of solubility brought about by the ionic crosslinking. Furthermore, among the three photosensitive ionomers investigated, mercurous copoly(vinyl alcohol-maleate) is the most sensitive and the best for the formation of a visible image. However, if the sensitivity for forming a relief image is considered the most important, mercurous copoly(vinyl alcohol-acrylate), which was reported in the previous paper, seems to have the best image-forming properties among the compounds tested.

Acknowledgement. The authors wish to thank Prof. Takahiro Tsunoda of Chiba University for allowing the use of the monochromator.

\section{REFERENCES}

1. T. Tsunoda, "Kankosei-jushi(Photopolymer)," Insatsu-gakkai Shuppan-bu, Tokyo, 1972, pp 1-2.

2. G. Brauer, "Handbook of preparative Inorganic Chemistry," P. G. Stecher, Translator, Academic Press Inc., London, 1965, pp 1120.

3. K. Sugita and S. Suzuki, Polymer J., 2, 283 (1971).

4. K. Sugita, M. Hase, and S. Suzuki, Bull. Chem. Soc. Japan, 45, 1921 (1972).

5. S. Suzuki and K. Sugita, Photogr. Sci. Eng., 15, 464 (1971). 